\section{Curvy, contoured hand instruments}

Acteon UK has just launched a new range of hand instruments called Bliss. They are strikingly different to most other instruments on the market. Curvy and contoured to maximise comfort, the first thing you'll notice is the urge to actually hold one. The second thing you'll notice is the soft silicone touch and the way it fits snugly in your working hand. You might even forget it's there because it is so lightweight, only $15 \mathrm{~g}$.

Bliss hand instruments have been designed by clinicians, working closely with the experienced manufacturers at Acteon. Not only is the handle shape new, but the tips have also been adjusted to maximise performance and improve access in hard to reach areas.

The pentagonal, flat-sided grip design is much more comfortable than typical cylindrical machined grips allowing for superior tactile feedback. The high-quality stainless steel used and the production process itself produces tips that are harder wearing and stay sharper for longer. The different instrument types are colour coded for easy recognition and can be personalised.

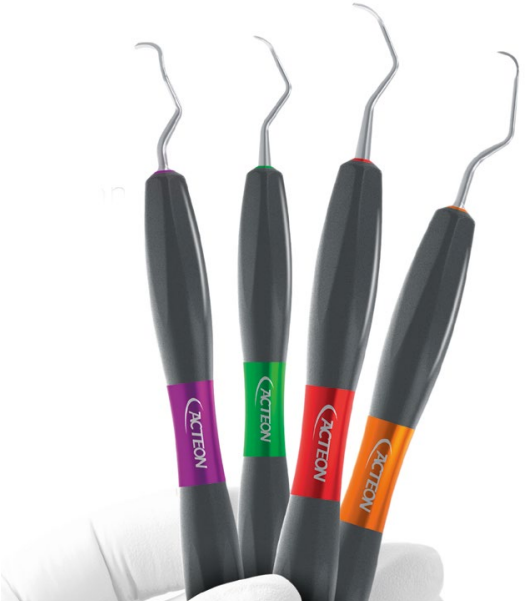

This combination of comfort, feel, hardness and sharpness ensures that users have to use less force, suffer less hand fatigue and patients experience a much enhanced, lighter and faster treatment.

Acteon UK specialises in the manufacture and supply of minimally invasive innovative technologies for dentists and dental professionals to diagnose, treat and educate patients efficiently.

For more information call 08000389840 (ext 1), email Marianne.maguire@acteongroup. com or visit acteongroup.com/uk-en

\title{
Now even more versatile and comfortable
}

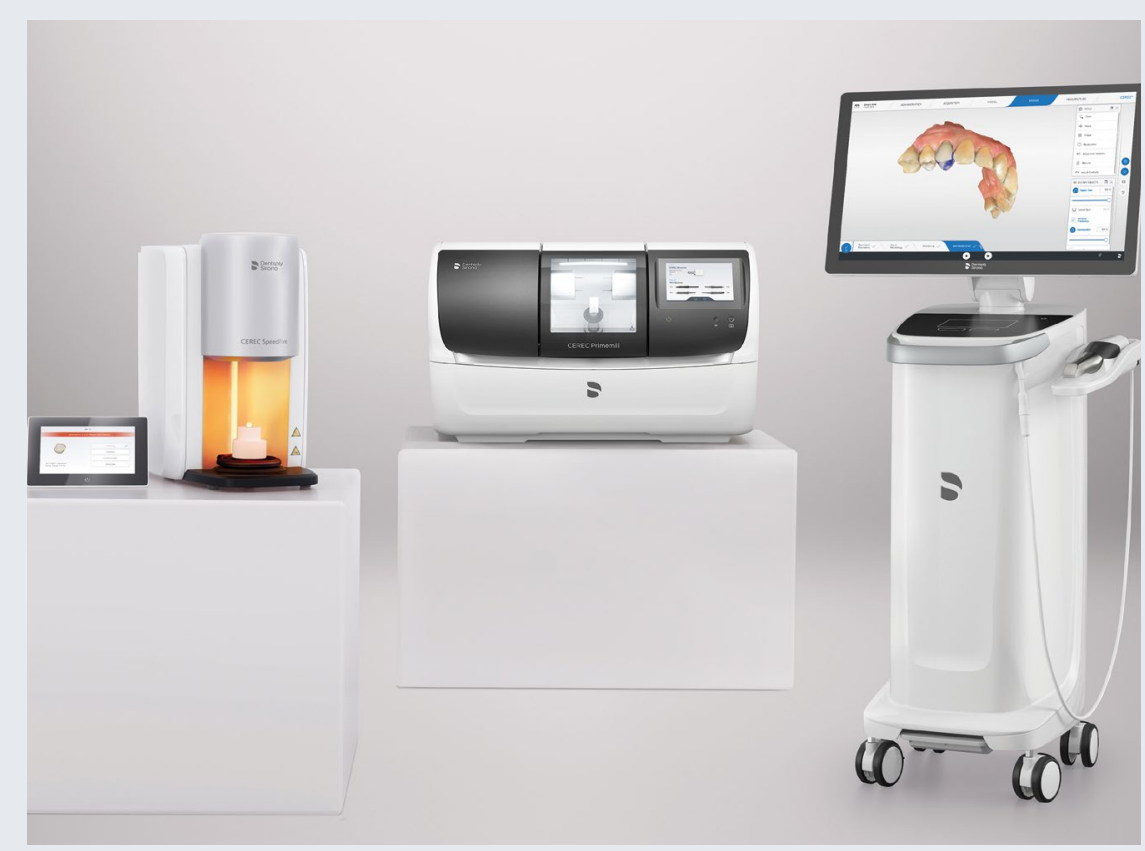

CEREC Primemill took the CEREC system to a new level of quality when it was launched one year ago: chairside dentistry is now faster, offers a wide range of materials, is easy to work with and ensures excellent results. CEREC users in around 50 countries are already taking advantage of the benefits of working with the new unit.

Dentsply Sirona recently introduced the CEREC SW 5.1.3 update, which includes 17 newly validated materials for the CEREC Primemill including PMMA for surgical guides and bridge blocks for grinded processes. With this update 49 different materials from nine different manufacturers are now validated for the unit. Restorations can now be made with ease from a wide range of materials including feldspar, hybrid and glass ceramics, lithium disilicate ceramics, zirconia, PMMA and composites.

Through these enhancements, the company has improved the ease of use, functionality, and stability - based on valuable insights gained from an intensive feedback exchange with the first users. The results were essentially achieved by updating the software. The update requires a pre-installed version of CEREC SW 5.1.2.

For new CEREC users, CEREC

Primemill, and in particular the zirconia workflow, represents an excellent start to the chairside treatment. Feedback provided by customers shows that CEREC Primemill helps advance treatment quality, particularly in terms of accuracy, speed and ease of use. Users report that from their experience they can arrange patient appointments differently because the workflow has become much faster. This is also supported by the fact that work steps that do not take place directly on the patient can be delegated. Ultimately, dentists gain more exclusive time for their patients to make them feel comfortable.

In addition to the developments in the clinical area, there are also advantages in profitability. These include saving time in the grinding and milling process itself compared to previously available units. With CEREC Primemill, restorations, especially those made of zirconia, can be milled even faster thanks to new tools and improved technology, which ultimately also has a time saving impact on the entire workflow. The ability to grind or mill restorations with high accuracy and precision, including the milling of zirconia crowns in Super Fast mode in as little as about five minutes, opens the door for even more efficient fabrication of ceramic restorations.

For more information visit www. dentsplysirona.com/cerecprimemill. 